ARTICLE

https://doi.org/10.1038/s41467-019-09007-1

\title{
Atomic insight into hydration shells around facetted nanoparticles
}

\author{
Sabrina L.J. Thomä ${ }^{1}$, Sebastian W. Krauss ${ }^{1}$, Mirco Eckardt', Phil Chater (i) ${ }^{2} \&$ Mirijam Zobel $^{1}$
}

Nanoparticles in solution interact with their surroundings via hydration shells. Although the structure of these shells is used to explain nanoscopic properties, experimental structural insight is still missing. Here we show how to access the hydration shell structures around colloidal nanoparticles in scattering experiments. For this, we synthesize variably functionalized magnetic iron oxide nanoparticle dispersions. Irrespective of the capping agent, we identify three distinct interatomic distances within $2.5 \AA$ from the particle surface which belong to dissociatively and molecularly adsorbed water molecules, based on theoretical predictions. A weaker restructured hydration shell extends up to $15 \AA$. Our results show that the crystal structure dictates the hydration shell structure. Surprisingly, facets of 7 and $15 \mathrm{~nm}$ particles behave like planar surfaces. These findings bridge the large gap between spectroscopic studies on hydrogen bond networks and theoretical advances in solvation science.

\footnotetext{
${ }^{1}$ Department of Chemistry, University of Bayreuth, Universitätsstr.30, 95440 Bayreuth, Germany. ${ }^{2}$ Diamond Light Source, Harwell Science \& Innovation Campus, Didcot, Oxfordshire OX11 ODE, UK. Correspondence and requests for materials should be addressed to M.Z. (email: mirijam.zobel@uni-bayreuth. de)
} 
S olvation shells have been shown to play a crucial role in many fields: at nano-bio interfaces ${ }^{1,2}$, for nanocrystals in molten inorganic salts ${ }^{3}$ or in the stabilization of $2 \mathrm{D}$ black phosphorous sheets in organic solvents ${ }^{4}$, or silica nanoparticles in ionic liquids ${ }^{5}$. Hydration forces have been accessed by atomic force microscopy $(\mathrm{AFM})^{6}$, and most recently frequency modulation AFM revealed molecular-level interfacial structures on $7 \mathrm{~nm}$ Si nanoparticles ${ }^{7}$. Hydration forces around nanoparticles deviate from the classical Derjaguin-Landau-Verwey-Overbeek (DLVO) theory of colloids, because the particles are comparable in size to the hydration shell and the nanoscopic forces are screened by hydration shells in which water molecules preferentially reorganize ${ }^{8}$. In order to understand the properties and interactions of hydration shells, atomistic insight into their structure is required. Over recent years, theoretical investigations into the importance of hydration shells has evolved considerably; molecular dynamics simulations have shown that at least two hydration layers are required to stabilise the structure of aqueous $\mathrm{Cu}_{7}$ clusters $^{9}$ and that the altered interfacial water structure impacts the adsorption and diffusion behaviour of ions to the surface $^{10}$ and slows down water dynamics ${ }^{11}$. Different diffusion components of the hydration layers around $\mathrm{SnO}_{2}$ and $\mathrm{TiO}_{2}$ nanoparticles had been extracted from quasi-elastic neutron scattering (QENS) experiments and were correlated to distinct hydration layers observed with molecular dynamics (MD) simulations ${ }^{12}$. The hydration layers at metal oxide surfaces differ, in general, from the bulk water properties and show modified dynamics and structure, typically varying within the individual layers. Spectroscopic techniques have revealed the modified strengths of hydrogen bond networks around nanoparticles via terahertz ${ }^{13,14}$ and $\mathrm{X}$-ray absorption spectroscopies ${ }^{15}$. X-ray reflectivity (XRR) can provide structural details of solvent layers at planar surfaces, but is also limited to the latter ${ }^{16}$. One of the significant remaining challenges is to gain experimental structural insight into the interfacial water structure in bulk solution. Recently, the X-ray pair distribution function (PDF) was used to prove the existence of solvation shells of organic solvent molecules around nanoparticles by observing electronic density oscillations which correlate with molecular reorientations ${ }^{17}$. As the molecular size of the solvent decreases down to that of water molecule, the requirements for the technique approach atomic resolution, which is commonly accepted to be challenging, if not impossible ${ }^{1,18}$. Several methods, including X-ray photoemission, $\mathrm{X}$-ray photoelectron spectroscopy and low energy electron diffraction can be used to probe the chemical and structural state of the very first adsorbed water layer, but provide no structural information on subsequent hydration shells.

The study of iron oxide nanoparticle (IONP) - water interfaces has a long history of experimental and theoretical investigations, since IONP are most frequently applied in the form of aqueous dispersions and as such are surrounded by bound water molecules. They are an important material in a range of fields spanning medicine as magnetic resonance contrast agents ${ }^{15,19,20}$, waste water treatment ${ }^{21}$ and catalysis ${ }^{22}$. Studies have shown that different facets induce differently adsorbed water layers around IONPs, i.e. for hematite $\mathrm{e}^{23,24}$ and magnetite ${ }^{25-28}$, confirmed by electron density profiles at selected crystallographic surface planes via $\mathrm{XRR}^{29}$. Bulk magnetite preferentially grows in (111) orientation $^{25,28}$, while $(110)^{27}$ and (001) planes are also energetically stable and occur ${ }^{30}$. Combined experimental and theoretical studies on water structures around magnetite or maghemite nanoparticles do not exist to our knowledge, and are sparse even for other industrially relevant material classes, such as hematite ${ }^{23}$, $\mathrm{TiO}_{2}{ }^{31}$, or $\mathrm{ZnS}^{32}$. The majority of the studies to date concern the adsorption of individual water molecules, dimers or trimers from the gas phase $\mathrm{e}^{18,25-28,33}$ and only few address multiple layers in a condensation regime to mimic bulk water surroundings ${ }^{23,31}$. Studies based on the adsorption of individual or dimeric water molecules have the drawback of overestimating interatomic distances $^{28}$.

Here, we show that we are able to resolve both the local interatomic distances in the adsorbed layer at the surface and the short-range ordered hydration shell out to $15 \AA$ around IONPs. We can allocate individual interatomic distances within the adsorbed layer to dissociatively and molecularly adsorbed water molecules at IONP facets, in good agreement with theoretical predictions ${ }^{25,28}$. This study therefore paves the way to greater atomistic structural insight in solvation science.

\section{Results}

Synthesis and characterization of IONPs. For this study, magnetic IONPs of 7 and $15 \mathrm{~nm}$ in size were synthesised via coprecipitation in basic solution at elevated temperature (for X-ray diffraction patterns, see Supplementary Figure 1). The IONPs were functionalized with 13 different small organic capping agents comprising dibutanoic acids, benzoic acids, basic amino acids, small $a$-hydroxy acids and some other bifunctional molecules (Supplementary Figure 2). IONP sizes were determined using transmission electron microscopy (TEM) (diameter from projected area, Supplementary Figure 3), dynamic light scattering (hydrodynamic diameter, Supplementary Table 1 and Supplementary Figure 4) and SAXS (particle size based on X-ray scattering contrasts, Supplementary Figure 4). TEM confirmed (100), (110) and (111) faceting of the particles (Supplementary Figure 5).

Availability of iron oxide surface. Thermogravimetric analysis (TGA) was performed to check for the availability of un-capped iron oxide surface which would be available to interact with water. Average ligand-surface coverages of $1-3.9 \mathrm{~nm}^{-2}$ were determined (only tartaric acid gave a higher coverage of $5.8 \mathrm{~nm}^{-2}$ ) in agreement with literature ${ }^{34}$ (Supplementary Figure 6, Supplementary Figure 7 and Supplementary Table 1.). According to Bixner et al. ${ }^{34}$, magnetite possess $3.5-5.9 \mathrm{~nm}^{-2}$ iron surface sites, thus leaving free iron oxide surface for interaction with water in this study. Moreover, water was shown to coexist with ligands on the particle surface even for fairly densely grafted IONP surfaces ${ }^{35}$.

PDF data evaluation. High-energy X-ray scattering data (65.4 $\mathrm{keV}$ ) were collected for stable colloidal IONP dispersions in water at ambient conditions and their corresponding backgrounds, from which we derived the PDFs, labelled $G(r)$ (Fig. 1a). The PDF is a real space representation of all interatomic distances present in the sample. Judicious subtraction of suitable backgrounds allows the distance contributions from individual sample constituents to be calculated. In order to highlight contributions from the hydration shell, all interatomic distances coming from the pure dry powder and the pure bulk water need to be subtracted. The subtraction of the water background from the dispersion (Supplementary Figure 8) and subsequent data correction and Fourier Transformation gives an experimental difference PDF (dPDF) which contains the contributions of the IONPs and their hydration shell (for details, see 'PDF data acquisition and processing' in Methods). Proper scaling of the experimental PDF of the, respectively, capped IONP powder to the d-PDFs allows its subsequent subtraction (Supplementary Figure 9). The resulting double-difference PDF (dd-PDF) contains only the signal from the hydration shell around the IONPs (Fig. 1b). In order to compare the hydration shells for the different capping agents, we additionally scaled the powder PDFs relatively to each other (Fig. 2a, see Methods for details, Supplementary Figure 10). 
a

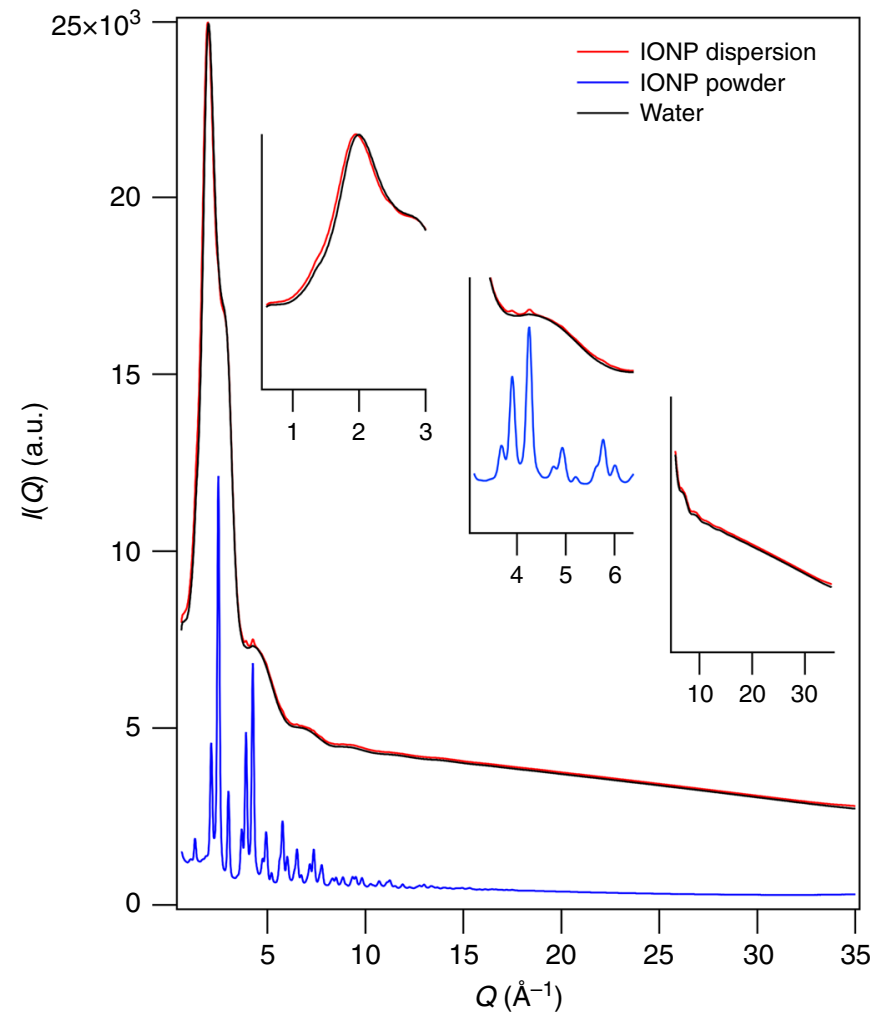

b

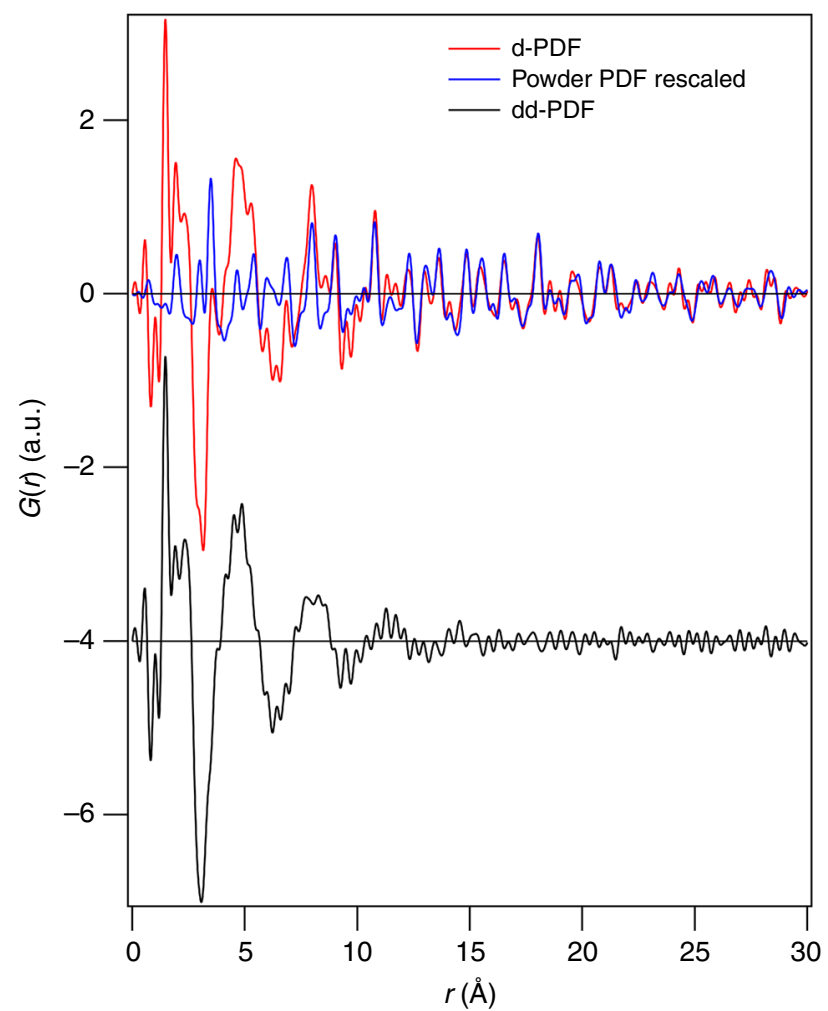

Fig. 1 Derivation of the hydration shell from experimental X-ray scattering data. a Integrated diffraction data $I(Q)$ for the arginine-capped iron oxide nanoparticle (IONP) dispersion (red), the water background (black) and the IONP powder (blue). b The arginine-capped powder pair distribution function (PDF) $(G(r)$, blue) is scaled to the difference PDF (d-PDF) (red) in the range of 15-30 $\AA$ as only IONP peaks are present there. The hydration shell (black, in offset) contains the structural information of the molecular arrangement of the water molecules around the IONPS

Figure 1b clearly features two regimes in the dd-PDF: sharp peaks below $3 \AA$ and a broad oscillation above $3 \AA$ (the small high-frequency ripples on this oscillation appear due to noise). The sharp peaks in the dd-PDF represent well defined interatomic distances, whereas broad oscillations reflect molecular reorientations. Water can adsorb in two possible ways at interfaces; dissociatively or molecularly. Moreover, the interfacial water regime most frequently features a complex structure consisting of a closely adsorbed water layer directly at the nanoparticle surface (sharp peaks in our case) and more loosely bound hydration layers (oscillations). The overall number of reoriented water layers in the hydration shell is thus the adsorbed layer plus the number of hydration layers.

\section{Discussion}

Two regimes, adsorption and hydration, are obvious and comparable for all ligands (Fig. 2a). The adsorption regime consists of three distinct interatomic distances with average values of 1.48 , 1.95 and $2.39 \AA$. The sinusoidal oscillation beyond represents the weaker bound hydration regime, decaying to the average bulk (random liquid) structure for distances $>15 \AA$. The three peak positions were directly read off the dd-PDFs and are listed in Table 1 for one representative of each group of capping agents (the full list is in Supplementary Table 2). The hydration layers can be modelled by an exponentially decaying sine wave with a wavelength of about $3 \AA$. The positions of the maxima of the hydration layers in Table 1 are retrieved from the fitted sine function. To our knowledge, the only MD study on IONP embedded in bulk water is by Spagnoli et al. ${ }^{24}$. Our experiment resulted in hydration layers with larger distances from the surface and a longer wavelength, which can be explained by a different crystal structure (magnetite vs hematite ${ }^{24}$ ) and an overestimated water density in ref. ${ }^{24}$. Strikingly, the peak positions in both the adsorbed and hydration layers are almost identical for all capping agents (Table 1). Only minor differences between the capping agents exist regarding amplitudes of both layers and are hypothesised to occur due to varied IONP concentrations (Supplementary Figure 12). The ligand groups demonstrate little or no impact on the hydration structure, suggesting that the crystallographic nature of the particles alone determines the hydration shell structure.

Bond length calculations from density functional theory (DFT) calculations of magnetite facets reported interatomic $\mathrm{Fe}-\mathrm{O}$ distances from dissociative and molecular adsorption which agree astonishingly well with our experimental evidence. For two different terminations of the $\mathrm{Fe}_{3} \mathrm{O}_{4}$ (111) facet, $\mathrm{Fe}-\mathrm{O}$ and $\mathrm{O}-\mathrm{H}$ distances of $1.47 \AA, 1.91 \AA$ and $2.32 \AA$ were predicted ${ }^{25,28}$.

In both DFT studies, water adsorbs dissociatively on both the $\mathrm{Fe}_{\mathrm{oct} 2}$ terminated surface with an $\mathrm{Fe}_{\mathrm{oct} 2}-\mathrm{O}_{\mathrm{OH}}$ distance of 1.907 $\AA^{25}$ and the $\mathrm{Fe}_{\text {tet } 1}$ site with a $\mathrm{Fe}_{\text {tet } 1}-\mathrm{O}_{\mathrm{OH}}$ bond of $2.32 \AA^{28}$. Both predicted distances are well in agreement with our observed peaks positions (1.89-1.96 and 2.33-2.40 $\AA$ ). Moreover, the $\mathrm{Fe}-\mathrm{O}_{\mathrm{H} 2 \mathrm{O}}$ distances for molecular adsorption are between $2.22 \AA$ on $\mathrm{Fe}_{3} \mathrm{O}_{4}$ (100) and 2.36-2.39 $\AA$ on different iron oxide hydroxides $(\alpha / \beta / \gamma$ $\mathrm{FeOOH})^{18,25,27}$. Thus, molecular adsorption accounts for our observed peak at $2.35 \AA$.

Our shortest distance of $1.48 \AA$ could be explained by hydrogen bonds formed as $\mathrm{O}_{\text {surface }}-\mathrm{H}_{\mathrm{H} 2 \mathrm{O}}$ bonds of $1.476 \AA^{28}$ or in-plane bonds between two molecularly co-adsorbed water molecules on the $\mathrm{Fe}_{\mathrm{oct} 2}$ site $\left(\mathrm{O}_{\mathrm{H} 2 \mathrm{O}}^{1 \mathrm{st}}-\mathrm{H}_{\mathrm{H} 2 \mathrm{O}}^{2 \mathrm{nd}}\right)$ with $1.462 \AA^{28}$. Other hydrogen bonds, which could occur due to dissociative adsorption of water 

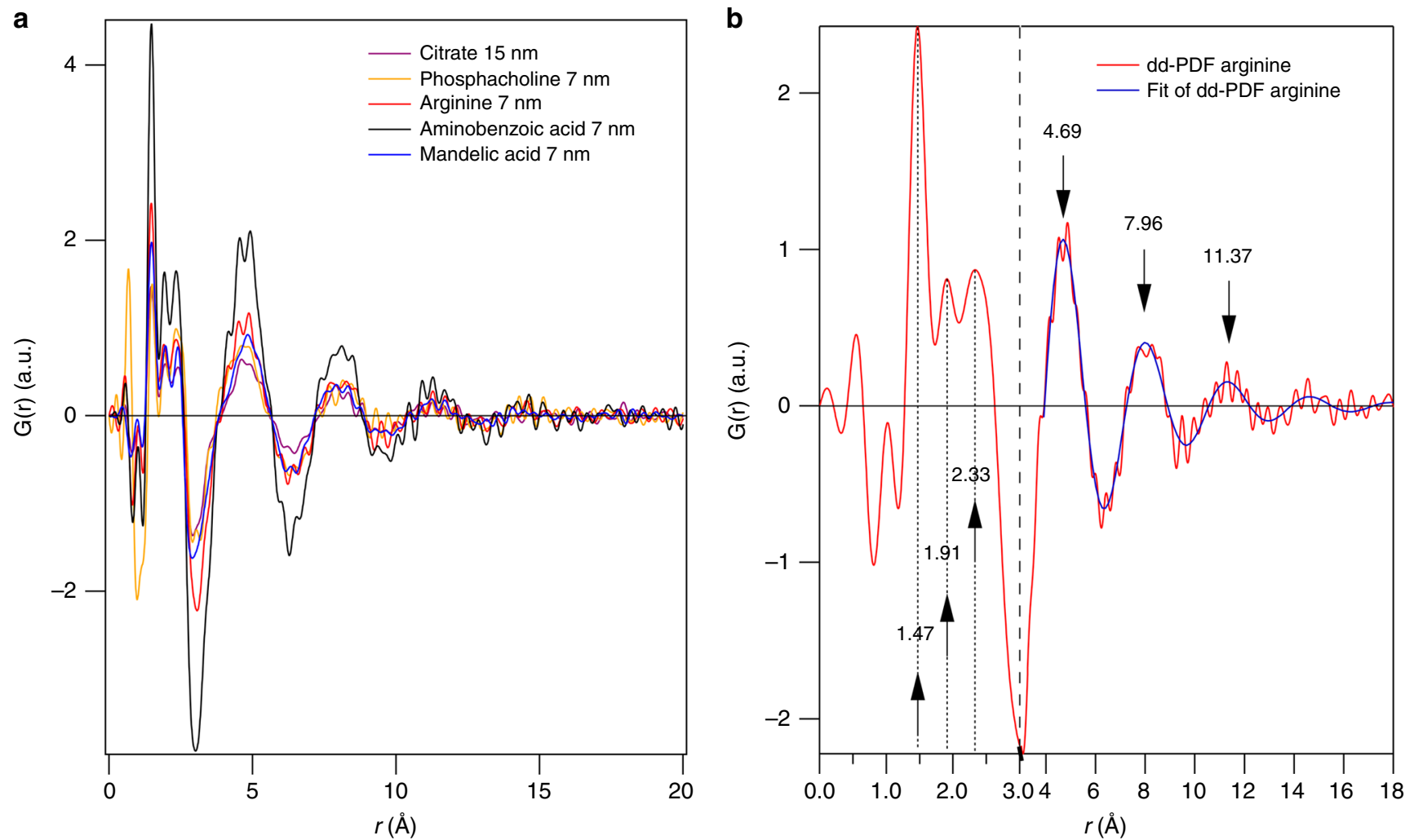

Fig. 2 Adsorbed water and hydration layers for various ligands. a Scaled hydration shells (double difference PDFs (dd-PDF)) of differently sized and functionalized iron oxide nanoparticles (IONPs) of selected samples (one capping agent of each group of Supplementary Figure 2; data of remaining sample shown in Supplementary Figure 11). b Scaled hydration shell of L-arginine-capped IONPs with its fit of a sinusoidal oscillation to weaker bound hydration shells at $4.69,7.96$ and $11.37 \AA$. The $x$-axis is split at $3 \AA$ and expanded for lower distances, in order two better show the three sharp peaks due to absorbed water at $1.47,1.91$ and $2.33 \AA$

Table. 1 Maxima positions of adsorbed and hydrated water layers

\begin{tabular}{|c|c|c|c|c|c|c|}
\hline Citrate $15 \mathrm{~nm}$ & 1.47 & 1.95 & 2.40 & 4.69 & 7.96 & 11.24 \\
\hline Arginine $7 \mathrm{~nm}$ & 1.47 & 1.91 & 2.33 & 4.69 & 7.96 & 11.24 \\
\hline Aminobenzoic acid $7 \mathrm{~nm}$ & 1.47 & 1.93 & 2.34 & 4.69 & 7.96 & 11.37 \\
\hline Mandelic acid $7 \mathrm{~nm}$ & 1.48 & 1.96 & 2.39 & 4.69 & 7.96 & 11.24 \\
\hline Average value & 1.48 & 1.95 & 2.39 & 4.70 & 7.96 & 11.27 \\
\hline
\end{tabular}

Peak positions of the first three sharp peaks due to absorbed water and the first three maxima of the oscillations in the hydration regime for selected samples (one capping agent of each group of Supplementary Figure 2). The average values, calculated from maxima positions of all 15 investigated samples (see Supplementary Table 2) are included for comparison, as well as the literature values to which we attribute the observed peak and maxima positions.

onto the surface are $\mathrm{O}_{\text {surface } \mathrm{OH}^{-}} \mathrm{H}_{\mathrm{H} 2 \mathrm{O}}$ and $\mathrm{H}_{\text {surface }} \mathrm{OH}^{-} \mathrm{O}_{\mathrm{H} 2 \mathrm{O}}$. In literature, no reference values for bond distances to surface hydroxyls were found, yet those bonds could be present on our surface and might contribute to the height of the first adsorption peak. One way to reason the high intensity of this first peak is the overall higher occurrence of hydrogen bonds in the interfacial region, although absolute amplitude of peaks at these small distances shall be treated with care given the minute difference signal from which the dd-PDFs are derived. Recent studies pointed to the fact that cooperative bonding of water clusters with shortened bond distances at interfaces preferentially occurs ${ }^{36}$. Furthermore, the influence and possibility of higher coordination numbers of edges, steps and corners, as well as the general modification of the hydrogen bond network around nanoparticles is not yet understood $^{11,15}$. Figure 3 schematically depicts the allocation of the individual PDF peaks to distinct interatomic distances in the first adsorption layers around facetted magnetite IONPs.

It is perhaps counterintuitive that nanoparticles with diameters of only $7 \mathrm{~nm}$ feature a hydration shell signature similar to bulk surfaces ${ }^{10,25}$. Spagnoli et al. studied the effect of particle size (1.6 and $2.7 \mathrm{~nm}$ ) and shape (facetted and spherical) via MD for hematite nanoparticles ${ }^{24}$. They suggested that already facetted $2.7 \mathrm{~nm}$ IONPs induce a well pronounced hydration structure with well separated interatomic distances within the adsorbed layer. The more disordered water structure at edges and corners does not play a major role any more. The magnetic $7 \mathrm{~nm}$ IONPs studied here feature (100), (110) and (111) facets (Supplementary Figure 5) and it is striking to observe how well theoretical 


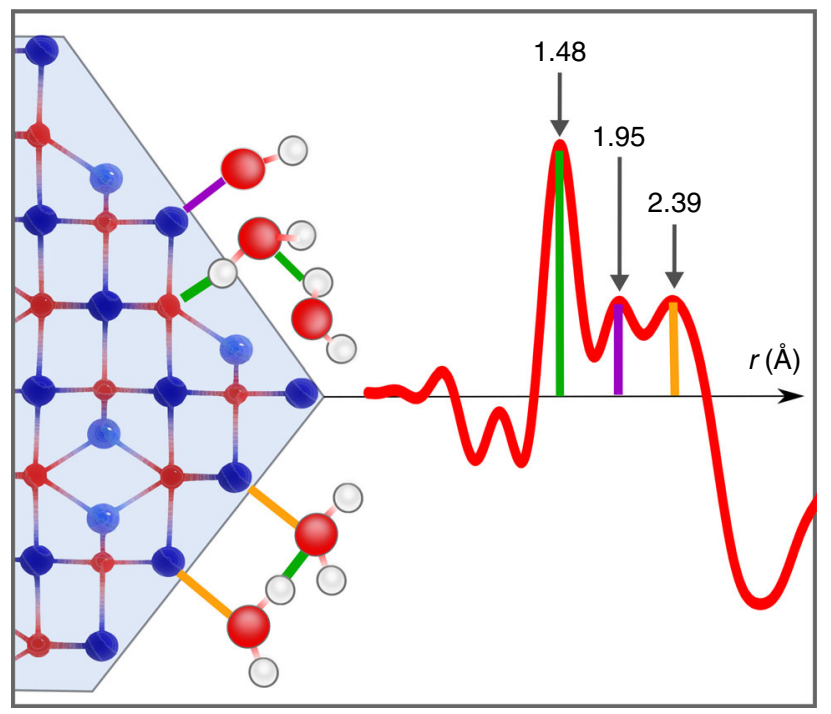

Fig. 3 Allocation of interatomic distances in adsorbed water layer. Facetted iron oxide nanoparticle surface with dissociatively and molecularly adsorbed water molecules and experimental signal from hydration shell. Oxygen atoms are red, hydrogen white, $\mathrm{Fe}_{\text {oct }}$ dark blue and $\mathrm{Fe}_{\text {tet }}$ light blue. Bonds resulting in the peaks at $1.48,1.95$ and $2.39 \AA$ are highlighted with solid lines in green, purple and yellow, respectively. The peak at $1.48 \AA$ belonging to hydrogen bonds can be allocated multiple times within the first adsorbed layer as indicated in the scheme. The scheme is a projection of a 3D adsorption structure onto 2D for illustration of relevant distances and thus distances and atom positions are distorted

predictions on the dominance of facets on water restructuring match this experimental work herein.

In order to assess whether the water structure observed for 7 $\mathrm{nm}$ particles was mainly due to planar facets, larger particles of $15 \mathrm{~nm}$ in diameter were synthesised with two different capping agents; citrate and malic acid. The observed hydration shells did not change with IONP size (Fig. 4). Ongoing studies will focus on the investigation of smaller particles $(<7 \mathrm{~nm})$ to investigate the impact of higher concentrations of edges and corners.

In summary, this study provides a method of investigating the interfacial hydration structure around colloidal nanoparticles. Facetted IONPs of 7 and $15 \mathrm{~nm}$ in diameter feature planar-like water ordering and the dissociative and molecular water binding states can be clearly distinguished. The effects of edges and corners are expected to be significant only for even smaller particle diameters. The size of the organic capping agents had minimal impact on the hydration shell structure, while the impact of concentration remains to be determined. This X-ray scatteringbased method of elucidating hydration shell structure bridges the structural gap between spectroscopy and theory. The high-energy $\mathrm{X}$-rays used in this study can easily penetrate sample containers and in-operando chambers and its applicability is not limited to accessible absorption edges like several X-ray spectroscopic techniques. This study therefore paves the way forward for atomistic structural studies of solvation science with future applicability ranging from ambient conditions with varying $\mathrm{pH}$ in living systems (enzymes, nano drug carriers) to high temperature/ pressure regimes of industrial liquid-phase catalysis, likely providing finally insight into the debated role of solvent molecules in self-assembly processes and during particle formation.

\section{Methods}

Chemicals. Chemicals for the nanoparticle synthesis were purchased and used without further purification: ethanol absolut (VWR), diethylene glycol (99\%, Alfa Aesar), N-methyl-2,2'-iminodiethanol (Merck), sodium hydroxide pellets (Merck),

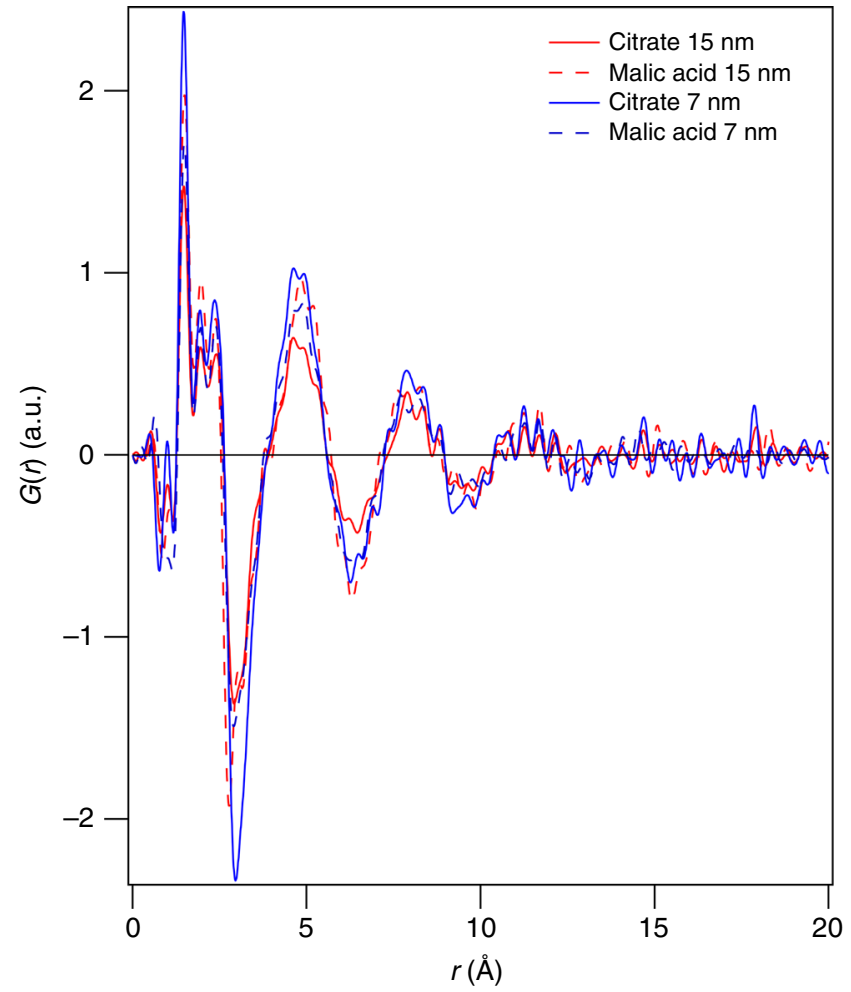

Fig. 4 Comparison of hydration shells for 7 and $15 \mathrm{~nm}$ iron oxide nanoparticles (IONPs). Double difference PDFs (dd-PDFs) of 7 (blue) and $15 \mathrm{~nm}$ (red) sized IONP with two different ligands; citrate (solid line) and malic acid (dashed line). The similarity of the dd-PDFs shows that the nanoparticle size does not influence the structure of the hydration shell

Hydrochloric acid solution (in water $1 \mathrm{M}$, Grüssing $\mathrm{GmbH}$ ), Sodium hydroxide solution (in water $1 \mathrm{M}$, Grüssing $\mathrm{GmbH}$ ), acetone (technical grade, Carl Roth $\mathrm{GmbH}$ ), iron(II) chloride tetrahydrate (Glentham Life Sciences) and iron(III) chloride hexahydrate (puriss. p.a. reag., $\geq 99 \%$, Sigma-Aldrich). Ligands for the nanoparticle modification were obtained from Sigma-Aldrich L-histidine monohydrochloride monohydrate ( $\geq 99 \%$ ), L-lysine monohydrochloride (BioUltra, $\geq 99.5 \%$ (AT)); 4-mercaptobenzoic acid (99\%); 4-aminobenzoic acid (Reagent Plus, $\geq 99 \%)$; DL-Lactic Acid ( $90 \%)$, phosphacholine chloride calcium salt tetrahydrate (Sigma grade); cysteamin (> 98\%); L-(+)-tartaric acid (99\%), from Alfa Aesar L-Arginine (98\%), L-(-)-malic acid (99\%), 4-hydroxybenzoic acid (99\%), ( \pm ) Mandelic Acid (99\%) and from Grüssing $\mathrm{GmbH}$ trisodium citrate dihydrate.

Nanoparticle synthesis. Water-dispersible IONPs with an approximate size of $7 \mathrm{~nm}$ and different ligands were obtained from hydrolysis of iron chelated complexes in diethylene glycol (DEG) according to Caruntu et al. . $^{37,38}$. IONPs with an approximate size of $15 \mathrm{~nm}$ were synthesized by performing the hydrolysis in a 70/ 30 mixture of DEG/ N-methyl-2,2'-iminodiethanol (NMDEA). 1 mmol FeCl 24 $\mathrm{H}_{2} \mathrm{O}(199 \mathrm{~g} / \mathrm{mol})$ and $2 \mathrm{mmol} \mathrm{FeCl}_{3} \cdot 6 \mathrm{H}_{2} \mathrm{O}(270 \mathrm{~g} / \mathrm{mol})$ were dissolved in $40 \mathrm{~g}$ DEG (DEG/NMDEA). A solution of $8 \mathrm{mmol} \mathrm{NaOH}(40 \mathrm{~g} / \mathrm{mol})$ in $20 \mathrm{~g}$ DEG (DEG/ NMDEA) was added. The reaction solution was then degassed under argon for about $2 \mathrm{~h}$ and subsequently heated to $220^{\circ} \mathrm{C}$ with a heating rate of $130^{\circ} \mathrm{C} / \mathrm{h}$. The temperature was kept constant for $2 \mathrm{~h}$ and then cooled to about $90^{\circ} \mathrm{C}$. At this point the reaction was divided in three parts (equal in volume). To each part a solution of $1 \mathrm{mmol}$ capping agent $(0.99-1.13)$ dissolved in $3 \mathrm{~mL} \mathrm{DEG} / \mathrm{H}_{2} \mathrm{O}$ was added. After the addition of the capping-agent-solutions the nanoparticle suspensions were stirred for another 10-15 min while cooling to room temperature. The next day the nanoparticle powder was precipitated by addition of acetone and isolated. For purification the powder was washed with absolute ethanol three times. The majority of the NP powder was dried in an oven at $80^{\circ} \mathrm{C}$. The rest was redispersed in $1.8 \mathrm{~mL}$ water (Milli-Q, or with addition of $\mathrm{NaOH} / \mathrm{HCl} 10 \mathrm{mM}$ ).

PDF data acquisition and processing. XRD measurements were carried out at $65.4 \mathrm{keV}(0.18957 \AA)$ at Diamond Light Source at beamline I15-1 (XPDF) using a Perkin Elmer detector XRD 4343 CT $\left(432 \times 432 \mathrm{~mm}^{2}\right.$ active area, $150 \times 150 \mu \mathrm{m}^{2}$ pixel size). Each data set was collected for a total of $6 \mathrm{~min} ; 12$ data collections of $30 \mathrm{~s}$ each were performed and then averaged. All samples were measured in $1 \mathrm{~mm}$ Kapton ${ }^{\circledast}$ capillaries. NIST silicon standards were used for distance calibration and instrumental resolution determination. Radial integration, masking and 
normalization of the data were provided by the beamline using automated processing in the DAWN software package ${ }^{39}$. PDF processing was carried out with ${ }_{x P D F s u i t e}{ }^{40}$ and fitting in IgorPro by WaveMetrics. The description of the hydration layers is based on an exponentially decaying sine wave according to Zobel et al. ${ }^{17}$ Besides the IONP dispersions, we measured the corresponding dry IONP powder for each ligand. All powders as well as the water data were measured under identical experimental conditions and treated in the same way during data analysis. The powder PDFs were scaled to the experimental d-PDFs with a scale factor $y_{1}$ in the distance range $>15 \AA$. The powder PDFs were scaled amongst each other for comparison of the hydration shells around differently functionalized IONPs with a scale factor $y_{2}$ (Supplementary Figure 10). For this we again used the intensity of the IONP PDF peak at $18.07 \AA$. All samples were scaled to an intensity of 0.5 at this point, since this value was the average value for all samples. The $15 \mathrm{~nm}$ particles were compared with the $7 \mathrm{~nm}$ particles by calculating PDFs in PDFgui for theoretical 7 and $15 \mathrm{~nm}$ magnetite NPs. The intensity ratio of the peak in the modelled PDF at $18.07 \AA$ for the 7 and $15 \mathrm{~nm}$ particles gave the factor by which we scaled up the experimental PDFs of $15 \mathrm{~nm}$ IONPs and corresponding dd-PDFs. Although the dd-PDFs feature high-frequency noise, e.g. see Fig. 1b, these can clearly be discerned from signal. When changing the $Q_{\max }$ used in the data processing, the high-frequency noise shifts in position, whereas the maxima of the three structural peaks $<3 \AA$ remain constant in position.

Transmission electron microscopy. TEM images of the nanoparticles for the evaluation of the particle size were acquired with a Zeiss Leo EM922 Omega TEM with an accelerating voltage of $200 \mathrm{kV}$. For each sample $4 \mu \mathrm{L}$ of very diluted NP dispersion were drop-casted on a carbon-coated meshed copper grid and dried in air. Aqueous dispersions were transferred to ethanol for faster drying. Particle sizes were obtained by measuring 100 particles from at least three images.

TEM images of the lattice planes of the nanoparticles (capped with L-lysine, aminobenzoic acid, tartaric acid and cysteamine) were acquired with a JEOL JEM$2200 \mathrm{FS}$ with an accelerating voltage of $200 \mathrm{kV}$ at high magnification. The samples were prepared as for evaluation of particle size. Occurring atomic distances were evaluated by creating diffraction patterns by Fourier Transformation with Image J and comparing those to projections of magnetite in Jmole. For each sample about 20 images were evaluated. The existence of the preferential growing facets of magnetite ((100), (111) and (110)) was confirmed (Extended Data Fig. 4).

Dynamic light scattering (DLS) and zeta potential. Evaluation of the hydrodynamic diameters and zeta potentials of the NPs was performed with a Particle Analyzer Litesizer 500 (Anton Paar, Germany) at $25^{\circ} \mathrm{C}$ in the automatic mode with a wavelength of $660 \mathrm{~nm}$. For each method three measurements were conducted and the average values with the standard deviation were calculated. The concentration of the NPs was adapted, so that the intensity was at least $300 \mathrm{kcounts} / \mathrm{s}$. Calculation of the number-weighted hydrodynamic radii was based on refractive indices and absorption coefficients from ${ }^{41}$. The zeta potential was calculated with the Smolouchowski approximation.

Thermal gravimetric analysis. TGA measurements were done from 30 to $500^{\circ} \mathrm{C}$ on a STA PT16000 (Linseis, Germany) with a heating ramp of $10 \mathrm{~K} / \mathrm{min}$ under argon atmosphere to prevent oxidation of IONPs. Ligand coverages were obtained from the mass loss above $160^{\circ} \mathrm{C}$, using particle diameters of 7 and $15 \mathrm{~nm}$.

\section{Data availability}

The data that support the findings of this study are available from the authors upon reasonable requests; please contact M.Z.

Received: 16 October 2018 Accepted: 8 February 2019

Published online: 01 March 2019

\section{References}

1. Nel, A. E. et al. Understanding biophysicochemical interactions at the nanobio interface. Nat. Mater. 8, 543-557 (2009).

2. Zhao, Z. et al. Nanocaged enzymes with enhanced catalytic activity and increased stability against protease digestion. Nat. Commun. 7, 10619 (2016).

3. Zhang, H. et al. Stable colloids in molten inorganic salts. Nature 542, 328-331 (2017).

4. Hanlon, D. et al. Liquid exfoliation of solvent-stabilized few-layer black phosphorus for applications beyond electronics. Nat. Commun. 6, 8563 (2015).

5. Gao, J., Ndong, R. S., Shiflett, M. B. \& Wagner, N. J. Creating nanoparticle stability in ionic liquid [C4mim] [BF4] by inducing solvation layering. ACS Nano 9, 3243-3253 (2015).

6. Israelachvili, J. N. Solvation forces and liquid structure, as probed by direct force measurements. Acc. Chem. Res. 20, 415-421 (1987).
7. Molino, P. J. et al. Hydration layer structure of biofouling-resistant nanoparticles. ACS Nano 12, 11610-11624 (2018).

8. Batista, C. A., Larson, R. G. \& Kotov, N. A. Nonadditivity of nanoparticle interactions. Science 350, 1242477 (2015).

9. Stenlid, J. H., Johansson, A. J., Kloo, L. \& Brinck, T. Aqueous solvation and surface oxidation of the Cu7nanoparticle: insights from theoretical modeling J. Phys. Chem. C 120, 1977-1988 (2016).

10. Kerisit, S., Cooke, D. J., Spagnoli, D. \& Parker, S. C. Molecular dynamics simulations of the interactions between water and inorganic solids. J. Mater. Chem. 15, https://doi.org/10.1039/b415633c (2005).

11. De La Pierre, M., Raiteri, P. \& Gale, J. D. Structure and dynamics of water at step edges on the calcite $\{10 \overline{1} 4\}$ surface. Cryst. Growth Des. 16, 5907-55914 (2016).

12. Mamontov, E. et al. Dynamics and structure of hydration water on rutile and cassiterite nanopowders studied by quasielastic neutron scattering and molecular dynamics simulations. J. Phys. Chem. C 111, 4328-4341 (2007).

13. Havenith-Newen, M., Schwaab, G. \& Sebastiani, F. Ion hydration and ion pairing as probed by $\mathrm{THz}$ spectroscopy. Angew. Chem. Int. Ed., https://doi. org/10.1002/anie.201805261 (2018).

14. Ebbinghaus, S. et al. An extended dynamical hydration shell around proteins. Proc. Natl Acad. Sci. USA 104, 20749-20752 (2007).

15. Petit, T. et al. Probing interfacial water on nanodiamonds in colloidal dispersion. J. Phys. Chem. Lett. 6, 2909-2912 (2015).

16. Magnussen, O. M. et al. X-ray reflectivity measurements of surface layering in liquid mercury. Phys. Rev. Lett. 74, 4444-4447 (1995).

17. Zobel, M., Neder, R. B. \& Kimber, S. A. Universal solvent restructuring induced by colloidal nanoparticles. Science 347, 292-294 (2015).

18. Meier, M. et al. Water agglomerates on $\mathrm{Fe}_{3} \mathrm{O}_{4}(001)$. Proc. Natl Acad. Sci. USA 115, E5642-E5650 (2018).

19. Ling, D. \& Hyeon, T. Chemical design of biocompatible iron oxide nanoparticles for medical applications. Small 9, 1450-1466 (2013).

20. Gupta, A. K. \& Curtis, A. S. Lactoferrin and ceruloplasmin derivatized superparamagnetic iron oxide nanoparticles for targeting cell surface receptors. Biomaterials 25, 3029-3040 (2004).

21. Mayo, J. T. et al. The effect of nanocrystalline magnetite size on arsenic removal. Sci. Technol. Adv. Mater. 8, 71-75 (2016).

22. Gawande, M. B., Branco, P. S. \& Varma, R. S. Nano-magnetite $\left(\mathrm{Fe}_{3} \mathrm{O}_{4}\right)$ as a support for recyclable catalysts in the development of sustainable methodologies. Chem. Soc. Rev. 42, 3371-3393 (2013).

23. Boily, J. F. et al. Thin water films at multifaceted hematite particle surfaces. Langmuir 31, 13127-13137 (2015).

24. Spagnoli, D., Gilbert, B., Waychunas, G. A. \& Banfield, J. F. Prediction of the effects of size and morphology on the structure of water around hematite nanoparticles. Geochim. Cosmochim. Acta 73, 4023-4033 (2009).

25. Li, X. \& Paier, J. Adsorption of water on the $\mathrm{Fe}_{3} \mathrm{O}_{4}(111)$ surface: structures, stabilities, and vibrational properties studied by density functional theory. $J$. Phys. Chem. C 120, 1056-1065 (2016).

26. Tombacz, E. et al. Water in contact with magnetite nanoparticles, as seen from experiments and computer simulations. Langmuir 25, 13007-13014 (2009).

27. Kundu, T. K., Rao, K. H. \& Parker, S. C. Atomistic simulation studies of magnetite surface structures and adsorption behavior in the presence of molecular and dissociated water and formic acid. J. Colloid Interface Sci. 295, 364-373 (2006)

28. Zhou, C. et al. Density functional theory study of water dissociative chemisorption on the $\mathrm{Fe}_{3} \mathrm{O}_{4}(111)$ surface. J. Phys. Chem. C 114, 21405-21410 (2010).

29. Catalano, J. G., Fenter, P. \& Park, C. Water ordering and surface relaxations at the hematite (110)-water interface. Geochim. Cosmochim. Acta 73, 2242-2251 (2009).

30. Santos-Carballal, D., Roldan, A., Grau-Crespo, R. \& de Leeuw, N. H. A DFT study of the structures, stabilities and redox behaviour of the major surfaces of magnetite $\mathrm{Fe}(3) \mathrm{O}(4)$. Phys. Chem. Chem. Phys. 16, 21082-21097 (2014).

31. Fazio, G., Selli, D., Ferraro, L., Seifert, G. \& Di Valentin, C. Curved $\mathrm{TiO}_{2}$ nanoparticles in water: short (chemical) and long (physical) range interfacial effects. ACS Appl. Mater. Interfaces https://doi.org/10.1021/acsami.8b08172 (2018).

32. Khalkhali, M., Zeng, H., Liu, Q. \& Zhang, H. Structural evolutions of ZnS nanoparticles in hydrated and bare states. J. Phys. Chem. C 120, 7870-7884 (2016).

33. Ovcharenko, R., Voloshina, E. \& Sauer, J. Water adsorption and O-defect formation on $\mathrm{Fe}_{2} \mathrm{O}_{3}(0001)$ surfaces. Phys. Chem. Chem. Phys. 18, 25560-25568 (2016).

34. Bixner, O., Lassenberger, A., Baurecht, D. \& Reimhult, E. Complete exchange of the hydrophobic dispersant shell on monodisperse superparamagnetic iron oxide nanoparticles. Langmuir 31, 9198-9204 (2015).

35. Soler, M. A. G. et al. Study of molecular surface coating on the stability of maghemite nanoparticles. Surf. Sci. 601, 3921-3925 (2007).

36. Merte, L. R. et al. Water clustering on nanostructured iron oxide films. Nat. Commun. 5, 4193 (2014). 
37. Caruntu, D. et al. Synthesis of variable-sized nanocrystals of $\mathrm{Fe}_{3} \mathrm{O}_{4}$ with high surface reactivity. Chem. Mater. 16, 5527-5534 (2004).

38. Qu, H., Caruntu, D., Liu, H. \& O’Connor, C. J. Water-dispersible iron oxide magnetic nanoparticles with versatile surface functionalities. Langmuir 27, 2271-2278 (2011).

39. Filik, J. et al. Processing two-dimensional X-ray diffraction and small-angle scattering data in DAWN 2. J. Appl. Crystallogr. 50, 959-966 (2017).

40. Yang, X., Juhas, P., Farrow, C. L. \& Billinge, S. J. L. xPDFsuite: an end-to-end software solution for high throughput pair distribution function transformation, visualization and analysis. J. Appl. Crystallogr. (2013) arXiv:1402.3163 [cond-mat.mtrl-sci].

41. Polyanskiy, M. Refrective Index Database, https://refractiveindex.info/ (2018).

\section{Acknowledgements}

We acknowledge funding by the Deutsche Forschungsgemeinschaft (DFG, German Research Foundation) via SFB 840 and a scholarship of the Bavarian Academy of Sciences and Humanities. We thank Marco Schwarzmann for TEM and TGA support, and Kevin Ament for synthesis support. We further thank the Bavarian Polymer Institute (BPI) for access to the keylabs 'Electron and Optical Microscopy' and 'Mesoscale Characterization: Scattering Techniques', as well as Sabine Rosenfeldt for SAXS measurements. Beam time at I15-1 (XPDF), Diamond Light Source (EE17345-1, is gratefully acknowledged. All experimental raw data are stored at the Diamond Lightsource. For access, please contact P.C. (philip.chater@diamond.ac.uk).

\section{Author contributions}

S.T. synthesized all samples, carried out all characterization, data processing and contributed to the manuscript. S.K. analysed TEM images. M.E. provided chemical and beamtime support and commented on the manuscript. P.C. supported the beamtime and manuscript. M.Z. initiated and supervised the project, contributed to the data analysis and wrote the manuscript.

\section{Additional information}

Supplementary Information accompanies this paper at https://doi.org/10.1038/s41467019-09007-1.

Competing interests: The authors declare no competing interests

Reprints and permission information is available online at http://npg.nature.com/ reprintsandpermissions/

Journal peer review information: Nature Communications thanks Chris Garvey and the other anonymous reviewers for their contribution to the peer review of this work. Peer reviewer reports are available.

Publisher's note: Springer Nature remains neutral with regard to jurisdictional claims in published maps and institutional affiliations.

(c) (i) Open Access This article is licensed under a Creative Commons Attribution 4.0 International License, which permits use, sharing, adaptation, distribution and reproduction in any medium or format, as long as you give appropriate credit to the original author(s) and the source, provide a link to the Creative Commons license, and indicate if changes were made. The images or other third party material in this article are included in the article's Creative Commons license, unless indicated otherwise in a credit line to the material. If material is not included in the article's Creative Commons license and your intended use is not permitted by statutory regulation or exceeds the permitted use, you will need to obtain permission directly from the copyright holder. To view a copy of this license, visit http://creativecommons.org/ licenses/by/4.0/.

(C) The Author(s) 2019 